\title{
Limitation of the Gaussian approximation in beam-beam simulations
}

\author{
Kaoru Yokoya \\ High Energy Accelerator Research Organization (KEK), Oho 1-1, Tsukuba-shi, Ibaraki-ken, Japan
}

(Received 25 September 2000; published 5 December 2000)

\begin{abstract}
The soft-Gaussian approximation is often employed in computer simulations of strong-strong beambeam interactions in storage rings. Its defect on the coherent oscillation frequency is pointed out and a possible remedy using Hermitian polynominals is discussed.
\end{abstract}

PACS numbers: 29.20.Dh, 29.27.-a

\section{INTRODUCTION}

The beam-beam interaction in storage rings is one of the subjects of beam physics to which analytic theories cannot provide sufficiently useful information for actual machines. In particular, for the strong-strong phenomena we have to use computer simulations. Although full details of interaction should in principle be obtained by the so-called particle-in-cell (PIC) simulation, it is very time consuming and suffers from numerical noises due to the finite number of macroparticles and to the finite mesh size.

Soft-Gaussian approximation is often used instead of PIC in order to overcome these problems. When particles come to a collision point, their center-of-mass position and the rms beam size are computed and the real beam-beam force is approximated by the force from the Gaussian charge distribution having the computed position and the rms size. This approximation greatly reduces the computation noise and the computing time.

However, the question of whether the soft-Gaussian approximation can replace PIC has not yet been answered systematically. Obviously, the soft-Gaussian approximation cannot express complicated distribution which might be encountered in extremely strong interactions well above the beam-beam limit, but they are not practically important for normal operations of colliders. In this report we consider an aspect of soft-Gaussian approximation in relatively weak cases and compare it with analytic theories.

The strength of beam-beam interaction is characterized by the parameter $\xi$ defined by

$$
\xi_{i}=\frac{\beta_{i}}{2 \pi \gamma} \frac{N r_{p}}{\sigma_{i}\left(\sigma_{x}+\sigma_{y}\right)}, \quad(i=x, y),
$$

where $\beta_{i}$ and $\gamma$ are the beta function and the Lorentz factor of the relevant beam, $N$ and $\sigma_{i}$ are the population and the beam size of the on-coming bunch, and $r_{p}$ is the classical radius of the particle (normally electron or proton). When $\xi$ is sufficiently small, the incoherent tune shift of a particle with small betatron amplitude is given by $\xi$.

On the other hand, the tune shift of the coherent oscillation ( $\pi$ mode) is given by

$$
\Delta \nu_{i}=\lambda \xi_{i}
$$

where the coefficient $\lambda$ is theoretically known to be slightly larger than unity and is dependent on the beam aspect ratio $R=\sigma_{y} / \sigma_{x}[1,2]$. More specifically, $\lambda_{\mathrm{h}}=1.33$ and $\lambda_{\mathrm{v}}=1.24$ for the horizontal and vertical oscillations of a flat beam $(R=0)$, respectively, and $\lambda_{\mathrm{r}}=1.21$ for a round beam. (In the following references, the "flat beam" does not mean a beam with zero height, but $R$ is sufficiently small.)

Many results of measurements and computer simulations are available about this factor $\lambda$.

An old result at KEK [3] agrees well with the theory for flat beams, and a recent result [4] also seems to agree although not very accurately. On the other hand, the value $\lambda_{\mathrm{v}}=1.1$ quoted in [5] at VEPP-2M contradicts the theory.

However, a comparison with measurements is not easy because the theory predicts the value only when $2 \pi \xi \ll 1$ (e.g., $\xi<0.01$, otherwise, the dynamic beta effect can easily change $\lambda$ by, say, 10\%), the bunch length is much shorter than the beta function, and the oscillation amplitude is much smaller than the beam size.

Because of this, a comparison with a strong-strong simulation is not easy either since $\xi<0.01$ requires a long-term tracking ( $\geq 10^{5}$ turns). Recent PIC simulations, however, can accurately compute the factor owing to the improvement of algorithms and computer speed. Ohmi [6] found a good agreement for horizontal oscillations of flat beams although $\xi$ is still large. Zorzano and Jones [7] found $\lambda_{\mathrm{r}}=1.2$, in good agreement with the theory, by using the so-called Hybrid-FMM algorithm for a small value of $\xi=0.0034$.

Zorzano [8] obtained $\lambda_{\mathrm{h}}=1.3$, which also agrees with the theory, by a totally different method, i.e., by solving the Vlasov equation numerically.

On the other hand, simulations by the soft-Gaussian approximation, which should be easier than PIC, seem to show smaller values systematically. The old results by Keil [9] are poor in statistics due to the limited computer power, but the curve for $\xi=0.01$ in Fig. 2 of [9] seems to show a value around $\lambda_{\mathrm{v}}=1.1$ (note that there are two collision points in his simulation). A much more accurate comparison is possible now owing to the computer improvements. In particular, the recent study of a detailed simulation for the Large Hadron Collider (LHC) by Zorzano and Zimmermann [10] quotes values between 1.09 and 1.15, depending on the aspect ratio. These values clearly disagree with the theory. Another soft-Gaussian simulation for the LHC 
by Furman [11] also shows a disagreement $\lambda_{\mathrm{r}}=1.1$. The above two studies adopt $\xi \approx 0.003$ for the LHC, which is small enough for comparison with the theory.

The first subject of the present report is this discrepancy between soft-Gaussian and PIC codes. It will be attributed to the nature of the soft-Gaussian approximation in Sec. II. Section III discusses a possible effect in the Landau damping due to this tune-shift error for the case of unequal-beam collision. Section IV studies an improvement of soft-Gaussian approximation by using the Hermitian polynomial.

\section{TUNE SHIFT OF COHERENT $\pi$ MODE IN SOFT-GAUSSIAN APPROXIMATION}

In this section we consider the frequency of smallamplitude coherent oscillation, analytically simulating the algorithm of the soft-Gaussian approximation.

Let us consider the simplest case when two counterrotating bunches having the same beam intensity and shape collide at one interaction point (IP) in the ring. We assume the two beams are electron and positron, but the conversion of the results to other species is obvious.

We normalize the horizontal/vertical coordinate and momentum by their unperturbed rms value and introduce the action-angle form

$$
x=\sqrt{2 J_{x}} \cos \phi_{x}, \quad p_{x}=-\sqrt{2 J_{x}} \sin \phi_{x}
$$

(similarly for $y$ ). Note that the canonical variables are $\left(J_{x}, \phi_{x}, R^{2} J_{y}, \phi_{y}\right)$ because of the different emittances in horizontal and vertical planes. We choose the machine azimuth $\theta$ as the independent variable. Then, the singleparticle motion of the positron is described by the Hamiltonian

$$
H=\nu_{x 0} J_{x}+\nu_{y 0} R^{2} J_{y}+V^{(-)}(x, y) .
$$

Here, $\nu_{x 0}$ and $\nu_{y 0}$ are the unperturbed tunes, and the potential $V^{(-)}$due to the electron beam is given by

$$
\begin{aligned}
V^{(-)}(x, y)= & \xi_{x}(1+R) \int d x^{\prime} d y^{\prime} \\
& \times \log \left[\left(x-x^{\prime}\right)^{2}+R^{2}\left(y-y^{\prime}\right)^{2}\right] \\
& \times \rho^{(-)}\left(x^{\prime}, y^{\prime}\right),
\end{aligned}
$$

where $R$ is the aspect ratio $\left(\sigma_{y}^{*} / \sigma_{x}^{*}\right)$ at the IP and $\rho$ is the distribution function of the electron normalized as $\int \rho d x d y=1$.

The phase-space distribution function consists of two parts, the unperturbed distribution $\Psi^{(0)}(\boldsymbol{J})$ and the oscillating part $\psi^{( \pm)}(\boldsymbol{J}, \boldsymbol{\phi})[\boldsymbol{J}, \boldsymbol{\phi}$ are the shorthand notations for $\left(J_{x}, J_{y}\right)$ and $\left.\left(\phi_{x}, \phi_{y}\right)\right]$. We approximate the unperturbed part by a Gaussian distribution

$$
\Psi^{(0)}(J)=\frac{1}{(2 \pi)^{2}} e^{-J_{x}-J_{y}} .
$$

The linearized Vlasov equation for the positron beam distribution $\psi^{(+)}$is written as

$$
\begin{aligned}
\frac{\partial \psi^{(+)}}{\partial \theta}+ & \nu_{x 0} \frac{\partial \psi^{(+)}}{\partial \phi_{x}}+\frac{\partial V^{(0)}}{\partial J_{x}} \frac{\partial \psi^{(+)}}{\partial \phi_{x}}- \\
& \frac{\partial V^{(0)}}{\partial \phi_{x}} \frac{\partial \psi^{(+)}}{\partial J_{x}}-\frac{\partial v^{(-)}}{\partial \phi_{x}} \frac{\partial \Psi^{(0)}}{\partial J_{x}}=0,
\end{aligned}
$$

where we omitted the terms related to the vertical oscillation. $V^{(0)}$ and $v^{( \pm)}$are the potentials coming from $\Psi^{(0)}$ and $\psi^{( \pm)}$, respectively,

$$
\begin{aligned}
V^{(0)}(x, y)= & \xi_{x}(1+R) \int \log \left[\left(x-x^{\prime}\right)^{2}+R^{2}\left(y-y^{\prime}\right)^{2}\right] \\
& \times \frac{e^{-\left(x^{\prime 2}+y^{\prime 2}\right) / 2}}{2 \pi} d x^{\prime} d y^{\prime}
\end{aligned}
$$

$$
\begin{aligned}
v^{( \pm)}(x, y)= & \xi_{x}(1+R) \int \log \left[\left(x-x^{\prime}\right)^{2}+R^{2}\left(y-y^{\prime}\right)^{2}\right] \\
& \times \psi^{( \pm)}\left(x^{\prime}, p_{x}^{\prime}, y^{\prime}, p_{y}^{\prime}\right) d x^{\prime} d p_{x}^{\prime} d y^{\prime} d p_{y}^{\prime} .
\end{aligned}
$$

We consider the horizontal dipole oscillation

$$
\psi^{( \pm)}=\operatorname{Re} \sum_{m} e^{i\left(m \phi_{x}-\nu \theta\right)} \psi_{m}^{( \pm)}(\boldsymbol{J})
$$

and take only the dipole mode $m=1$. Then Eq. (7) becomes

$$
\begin{aligned}
\left(\nu-\nu_{x 0}\right) \psi_{1}^{(+)}= & i \int \frac{d \boldsymbol{\phi}}{(2 \pi)^{2}} e^{-i \phi_{x}} \\
& \times\left\{-\frac{\partial V^{(0)}}{\partial J_{x}} \frac{\partial \tilde{\psi}^{(+)}}{\partial \phi_{x}}+\frac{\partial \tilde{\boldsymbol{v}}^{(-)}}{\partial \phi_{x}} \frac{\partial \Psi^{(0)}}{\partial J_{x}}\right\},
\end{aligned}
$$

where the tilde indicates that the factor $e^{-i \nu \theta}$ is to be taken off. The contribution of the first term in the curly brackets on the right-hand side is $\xi_{x} Q(J) \psi_{1}^{(+)}$where

$$
\begin{aligned}
Q(\boldsymbol{J})= & \frac{\partial}{\partial J_{x}} \int \frac{d \phi}{(2 \pi)^{2}} \frac{V^{(0)}}{\xi_{x}} \\
= & (1+R) \frac{\partial}{\partial J_{x}} \int \frac{d \phi}{(2 \pi)^{2}} \int d x^{\prime} d y^{\prime} \\
& \times \log \left[\left(x-x^{\prime}\right)^{2}+R^{2}\left(y-y^{\prime}\right)^{2}\right] \\
& \times \frac{e^{-\left(x^{\prime 2}+y^{\prime 2}\right) / 2}}{2 \pi} .
\end{aligned}
$$

Now consider the second term, where the soft-Gaussian approximation comes in. From the algorithm of the softGaussian approximation,

$$
v^{(-)}(x, y)=V^{(0)}\left(x-X^{(-)}, y\right)-V^{(0)}(x, y),
$$

where $X^{(-)}$is the center-of-mass position of the second beam,

$$
X^{(-)}=\int x \psi^{(-)} d \boldsymbol{J} d \boldsymbol{\phi}=e^{-i \nu \theta} \int x e^{i \phi_{x}} \psi_{1}^{(-)} d \boldsymbol{J} d \boldsymbol{\phi} .
$$




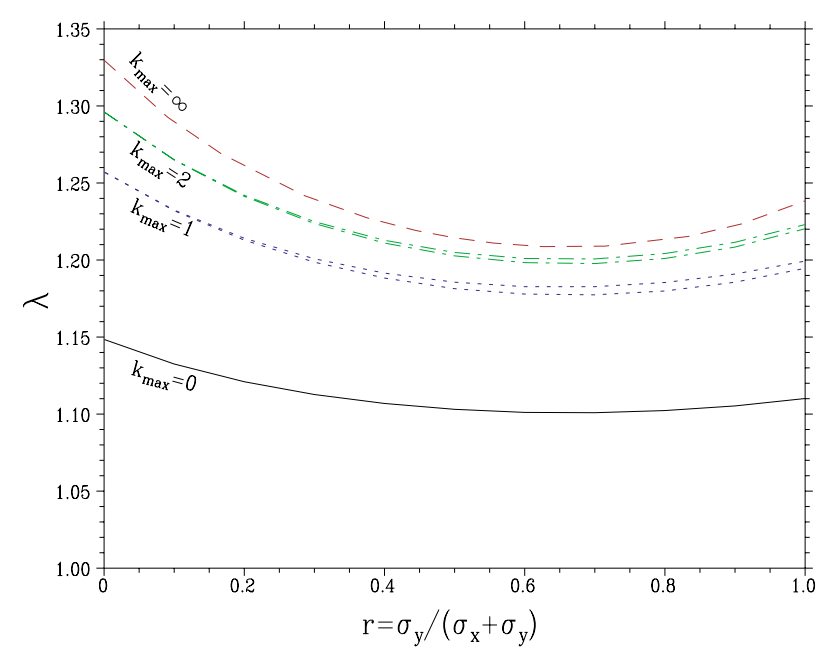

FIG. 1. (Color) $\pi$-mode eigenvalue as a function of the aspect ratio. $k_{\max }$ is the truncation of the Hermitian polynomial. $k_{\max }=0$ corresponds to the soft-Gaussian approximation and $k_{\max }=\infty$ is the exact theory.

(In actual soft-Gaussian approximation the rms size is also adjusted every turn, but this does not contribute to the infinitesimal dipole oscillation due to the symmetry.) Since we consider infinitesimal oscillation,

$$
\begin{gathered}
v^{(-)}(x, y)=-X^{(-)} \frac{\partial V^{(0)}}{\partial x} \\
X^{(-)}=2 \pi^{2} e^{-i \nu \theta} \int \sqrt{2 J_{x}} \psi_{1}^{(-)} d \boldsymbol{J} .
\end{gathered}
$$

After some manipulation, the contribution of the second term is found to be

$$
-\xi_{x} e^{-J_{x}-J_{y}} \int \sqrt{J_{x} J_{x}^{\prime}} Q(\boldsymbol{J}) \psi_{1}^{(-)}\left(\boldsymbol{J}^{\prime}\right) d \boldsymbol{J}^{\prime} .
$$

Thus, by introducing $f^{( \pm)}$defined by

$$
\psi_{1}^{( \pm)}(\boldsymbol{J})=e^{-\left(J_{x}+J_{y}\right) / 2} f^{( \pm)}(\boldsymbol{J})
$$

we obtain an integral equation

$$
\lambda f^{(+)}(\boldsymbol{J})=Q(\boldsymbol{J}) f^{(+)}(\boldsymbol{J})-\int G\left(\boldsymbol{J}, \boldsymbol{J}^{\prime}\right) f^{(-)}\left(\boldsymbol{J}^{\prime}\right) d \boldsymbol{J}^{\prime},
$$

where

$$
\lambda=\left(\nu-\nu_{x 0}\right) / \xi_{x}
$$

$$
G\left(\boldsymbol{J}, \boldsymbol{J}^{\prime}\right)=\sqrt{J_{x} J_{x}^{\prime}} e^{-\left(J_{x}+J_{y}+J_{x}^{\prime}+J_{y}^{\prime}\right) / 2} Q(\boldsymbol{J}) .
$$

The $\pi$ mode $f=f^{(+)}=-f^{(-)}$equation is

$$
\lambda f(\boldsymbol{J})=Q(\boldsymbol{J}) f(\boldsymbol{J})+\int G\left(\boldsymbol{J}, \boldsymbol{J}^{\prime}\right) f\left(\boldsymbol{J}^{\prime}\right) d \boldsymbol{J}^{\prime} .
$$

Since the kernel $G$ is degenerate (i.e., a product of a function of $\boldsymbol{J}$ and a function of $\boldsymbol{J}^{\prime}$ ), one can easily obtain an eigenvalue equation

$$
1=\int d \boldsymbol{J} \frac{Q(\boldsymbol{J})}{\lambda-Q(\boldsymbol{J})} J_{x} e^{-J_{x}-J_{y}} .
$$

The eigenvalue $\lambda$ is plotted in Fig. 1, where six curves are shown. The dashed curve at the top labeled $k_{\max }=\infty$ is the result from the exact theory, and the solid curve at the bottom labeled $k_{\max }=0$ is from the soft-Gaussian approximation (the other four curves are explained later).

One finds that the soft-Gaussian approximation gives a considerably different result from the exact theory. Interestingly, the soft-Gaussian eigenvalue is nearly at the middle between the exact value and that of the rigidGaussian model $(\lambda=1)$ [12] for any aspect ratio.

Numerical values are summarized in Table I for the three important cases, namely, $R=0$ (horizontal oscillation of very flat beams), $R=1$ (round beams), and $R=\infty$ (vertical oscillation of horizontally flat beams). The values obtained in an actual soft-Gaussian simulation [10] are listed in the bottom row, which is to be compared with the top row (the flat beam used in [10] is for $R=1 / 16$ ). They agree quite well with our theoretical values. The remaining small differences may be attributed to the dynamic beta effect. We can thus conclude that the discrepancy between soft-Gaussian simulations and the simple theory is due to the nature of the Gaussian form.

The physical reason for the difference is the following. Figure 2 schematically shows the beam profile during coherent oscillation. The dotted curve is the equilibrium distribution and the dashed curve is the real distri-

\begin{tabular}{|c|c|c|c|c|}
\hline & $k_{\max }$ & $\begin{array}{c}R=0 \\
\lambda_{\mathrm{h}}\end{array}$ & $\begin{array}{c}\text { Aspect ratio } \\
R=1 \\
\lambda_{\mathrm{r}} \\
\end{array}$ & $\begin{array}{c}R=\infty \\
\lambda_{\mathrm{v}}\end{array}$ \\
\hline Soft-Gaussian & 0 & 1.148 & 1.103 & 1.110 \\
\hline Third-order Hermitian & 1 & $1.257(1.257)$ & $1.181(1.186)$ & 1.195 (1.199) \\
\hline Fifth-order Hermitian & 2 & $1.296(1.296)$ & $1.203(1.205)$ & $1.220(1.223)$ \\
\hline Exact theory & $\infty$ & 1.330 & 1.214 & 1.238 \\
\hline Simulation [10] & & 1.15 & 1.10 & 1.09 \\
\hline
\end{tabular}
bution in oscillation. One sees only the core oscillates, as found in the theory (Fig. 1 in [2]) and simulation (Fig. 3 in [10]). When this profile is approximated by a Gaussian form shown by the solid curve, the oscillation amplitude of the core becomes smaller, which underestimates the beam-beam restoring force. Thus, the eigenvalue appears smaller.

TABLE I. Eigenvalues for the equal-beam case. 


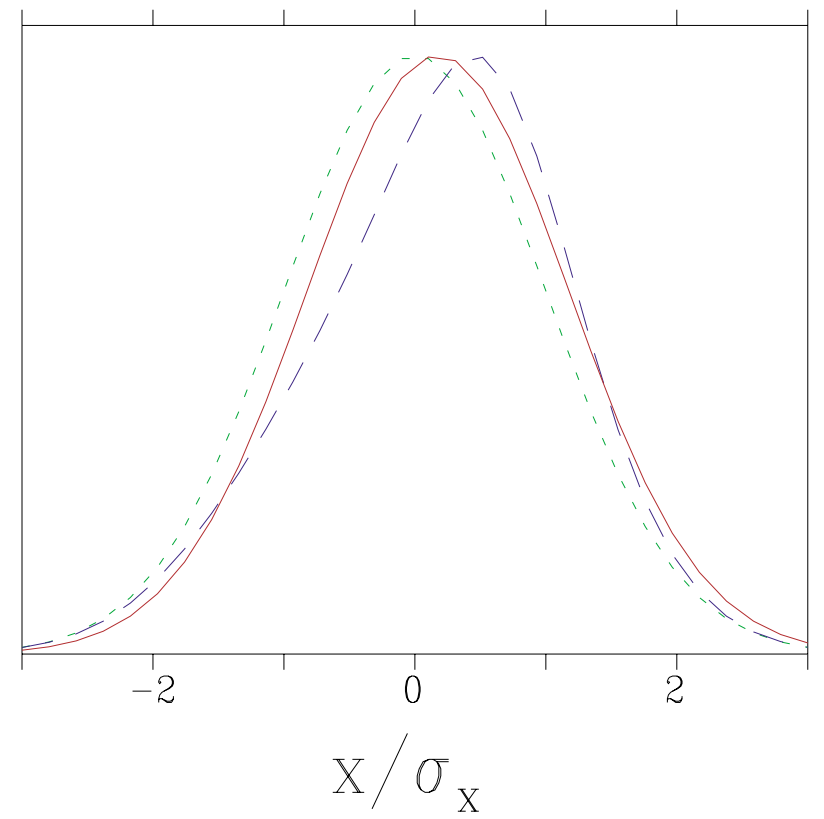

FIG. 2. (Color) Beam profile during coherent oscillation. The horizontal axis is the horizontal coordinate $x$. The dotted curve is the equilibrium distribution, the dashed curve the real distribution in oscillation, and the solid curve its Gaussian approximation.

\section{LANDAU DAMPING}

As we have seen in the previous section, the distance between the $\pi$-mode frequency and the edge of the incoherent frequency band $0<\lambda<1$ (continuum) in softGaussian simulation appears to be about half of what it should be. A question may arise as to whether softGaussian simulation correctly expresses the Landau damping, which is expected when a mode frequency falls in the incoherent frequency band. This is not an easy question because the Landau damping does not necessarily occur near the edge of the band, but whether the mode goes into the band is still a good criterion for the damping to occur.

In this section we consider a collision of beams of unequal intensity (but the same beam size). A new parameter is the ratio of the beam-beam parameters $\xi^{(-)} / \xi^{(+)}$ (we take $\xi^{(-)} \leq \xi^{(+)}$). In the limit $\xi^{(-)} / \xi^{(+)} \rightarrow 0$, there can be no coherent oscillation because it is the weak-strong case. It is known [13] that the $\pi$ mode goes into the continuum when the ratio of the beam-beam parameter $\xi^{(-)} / \xi^{(+)}$ is less than about 0.6 for round beams.

The integral equation is easily obtained from Eq. (19):

$$
\begin{aligned}
& \frac{\nu-\nu_{x 0}}{\xi_{x}^{(+)}} f^{(+)}=Q f^{(+)}-G \circ f^{(-)}, \\
& \frac{\nu-\nu_{x 0}}{\xi_{x}^{(-)}} f^{(-)}=Q f^{(-)}-G \circ f^{(+)},
\end{aligned}
$$

where $\circ$ indicates the integral operation with the kernel $G$. The eigenvalue equation is

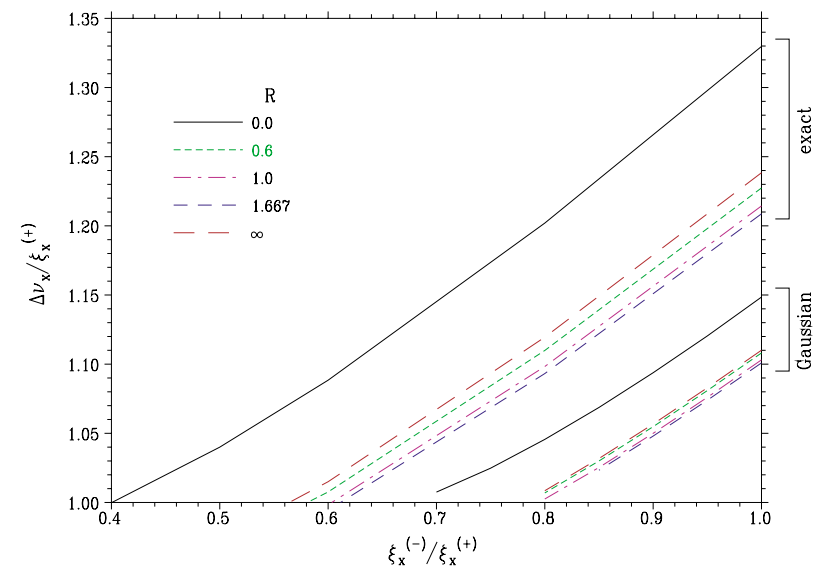

FIG. 3. (Color) $\pi$-mode eigenvalue as a function of the intensity ratio $\xi^{(-)} / \xi^{(+)}$for various values of the aspect ratio.

$$
\begin{aligned}
\int_{0}^{\infty} d \boldsymbol{J} \frac{Q(\boldsymbol{J}) J_{x} e^{-J_{x}-J_{y}}}{\left(\nu-\nu_{x 0}\right) / \xi_{x}^{(+)}-Q(\boldsymbol{J})} \times \\
\int_{0}^{\infty} d \boldsymbol{J} \frac{Q(\boldsymbol{J}) J_{x} e^{-J_{x}-J_{y}}}{\left(\nu-\nu_{x 0}\right) / \xi_{x}^{(-)}-Q(\boldsymbol{J})}=1 .
\end{aligned}
$$

When $\xi^{(-)}<\xi^{(+)}, \nu$ can go into the continuum of the positron beam (weaker beam). Therefore, Landau damping is expected when $\lambda \equiv\left(\nu-\nu_{x 0}\right) / \xi_{x}^{(+)}<1$.

The eigenvalue is plotted in Fig. 3 as a function of the intensity ratio $\xi^{(-)} / \xi^{(+)}$for various values of the aspect ratio. The upper group of curves is the result of the exact theory and the lower group the soft-Gaussian approximation. One finds that, in the round beam case, for example, the $\pi$ mode goes into the continuum at $\xi^{(-)} / \xi^{(+)} \sim 0.8$ in the soft-Gaussian approximation.

\section{HERMITIAN POLYNOMIAL EXPANSION}

As we saw in Sec. II, the reason for the disagreement in the tune shift is that the Gaussian approximation cannot take into account asymmetric distribution. A natural extension from the Gaussian model as a remedy to this disagreement is to include higher order moments.

Consider a Gaussian distribution with rms size $\left(\sigma_{x}, \sigma_{y}\right)$

$$
\rho_{G}\left(x, y ; \sigma_{x}, \sigma_{y}\right)=\frac{1}{2 \pi \sigma_{x} \sigma_{y}} e^{-x^{2} / 2 \sigma_{x}^{2}-y^{2} / 2 \sigma_{y}^{2}},
$$

and denote its potential by $\Phi_{G}(x, y)$. The latter satisfies the two-dimensional Poisson equation $\Delta \Phi_{G}=\rho_{G}$. In actual simulation the derivative of $\Phi_{G}$ is needed, which can be expressed using the complex error function [14].

When a distribution is expanded with Hermitian polynomials as

$$
\rho(x, y)=\sum_{k, l} a_{k, l} H_{k}\left(x / \sigma_{x}\right) H_{l}\left(y / \sigma_{y}\right) \rho_{G}\left(x, y ; \sigma_{x}, \sigma_{y}\right),
$$


where the Hermitian polynomial is defined by

$$
H_{n}(x)=(-1)^{n} e^{x^{2} / 2} \frac{d^{n}}{d x^{n}} e^{-x^{2} / 2},
$$

the corresponding potential is given by

$$
\Phi=\sum_{k, l}(-1)^{k+l} a_{k, l} \sigma_{x}^{k} \sigma_{y}^{l} \frac{\partial^{k}}{\partial x^{l}} \frac{\partial^{l}}{\partial y^{l}} \Phi_{G},
$$

and, therefore, the force is also computed from derivatives of the complex error function (the derivatives can also be represented by the complex error function).

Owing to the orthogonality of the Hermitian polynomials, the coefficient $a_{k, l}$ can be computed from $\rho$ by

$$
a_{k, l}=\frac{1}{k ! l !} \int H_{k}\left(x / \sigma_{x}\right) H_{l}\left(y / \sigma_{y}\right) \rho(x, y) d x d y,
$$

and, when the distribution is given in the form of macroparticle coordinates $\left(x_{j}, y_{j}\right)(j=1,2, \ldots, N)$,

$$
a_{k, l}=\frac{1}{k ! l !} \frac{1}{N} \sum_{j=1}^{N} H_{k}\left(x_{j} / \sigma_{x}\right) H_{k}\left(y_{j} / \sigma_{y}\right) .
$$

There are possible variations of Hermitian expansion with respect to the treatment of the first two moments.
Usually, in the soft-Gaussian approximation, the distribution is approximated by $\rho_{G}\left(x-X, y-Y ; \sigma_{x}, \sigma_{y}\right)$. If one starts expansion from this form, the first two coefficients $a_{k, l}$ vanish. But, instead, we can use the initial origin and the initial beam size as the base Gaussian distribution. In the latter case, the center-of-mass position and the rms size are represented by $a_{10}, a_{01}, a_{20}$, and $a_{02}$. These two different ways of expansion give different results in actual simulations, but they are equivalent when one considers infinitesimal oscillation analytically (e.g., $e^{-\left(x-a_{10}\right)^{2} / 2}$ and $\left(1+a_{10} x\right) e^{-x^{2} / 2}$ are equivalent if $a_{10}$ is infinitesimal). In actual simulations the former will be better for expressing finite-amplitude oscillations and considerable amount of beam size change. In this report we adopt the latter form for mathematical simplicity.

Now, let us go back to Eq. (11) and compute the coherent tune in the Hermitian expansion model. The perturbed potential $v^{(-)}$is given by

$$
\tilde{\boldsymbol{v}}^{(-)}(x, y)=\sum_{(k, l) \neq(0,0)}^{\infty}(-1)^{k+l} \tilde{a}_{k, l}^{(-)} \frac{\partial^{k}}{\partial x^{k}} \frac{\partial^{l}}{\partial y^{l}} V^{(0)}(x, y)
$$

and the coefficients $\tilde{a}_{k, l}^{(-)}$by

$$
\begin{aligned}
\tilde{a}_{k . l} & =\frac{1}{k ! l !} \int_{-\infty}^{\infty} H_{k}(x) H_{l}(y) \tilde{\rho}^{(-)}(x, y) d x d y \\
& =\frac{1}{k ! l !} \int_{-\infty}^{\infty} H_{k}\left(\sqrt{2 J_{x}} \cos \phi_{x}\right) H_{l}\left(\sqrt{2 J_{y}} \cos \phi_{y}\right) \cos \left(\phi_{x}\right) \psi_{1}^{(-)} d \boldsymbol{\phi} d \boldsymbol{J} .
\end{aligned}
$$

Because of the even-odd property of $H_{n}$, only odd $k$ and even $l$ contribute to the horizontal dipole oscillation. Thus,

$$
\tilde{a}_{2 k+1,2 l}=\frac{1}{2}(2 \pi)^{2} \sqrt{2 J_{x}} \int d \boldsymbol{J} L_{k, l}(\boldsymbol{J}) \psi_{1}^{(-)}(\boldsymbol{J}),
$$

where $L_{k, l}(\boldsymbol{J})$ is a known polynomial of $\boldsymbol{J}$ (see the Appendix).

Putting Eq. (33) into Eq. (11), one finds the contribution of the second term on the right-hand side to be

$$
-\xi_{x} e^{-J_{x}-J_{y}} \int d \boldsymbol{J}^{\prime} \sqrt{J_{x} J_{x}^{\prime}} \sum_{k, l=0}^{\infty} K_{k, l}(\boldsymbol{J}) L_{k, l}\left(\boldsymbol{J}^{\prime}\right) \psi_{1}^{(-)}\left(\boldsymbol{J}^{\prime}\right),
$$

where

$$
K_{k, l}(\boldsymbol{J})=\frac{\partial}{\partial J_{x}} \int \frac{d \phi_{x} d \phi_{y}}{(2 \pi)^{2}} \frac{\partial^{2 k}}{\partial x^{2 k}} \frac{\partial^{2 l}}{\partial y^{2 l}} \frac{V^{(0)}(x, y)}{\xi_{x}} .
$$

$K_{k, l}(\boldsymbol{J})$ is a complicated function of $\boldsymbol{J}$ and has to be computed by numerical integration except for the case $R=0$ (see the Appendix). In any case, $K_{0,0}(\boldsymbol{J})=Q(\boldsymbol{J})$.

Thus, again using $f$ defined in Eq. (18), we finally obtain an integral equation

$$
\begin{aligned}
\lambda f^{(+)}(\boldsymbol{J})= & Q(\boldsymbol{J}) f^{(+)}(\boldsymbol{J}) \\
& -\sum_{k, l=0}^{\infty} \int d \boldsymbol{J}^{\prime} G_{k, l}\left(\boldsymbol{J}, \boldsymbol{J}^{\prime}\right) f^{(-)}\left(\boldsymbol{J}^{\prime}\right),
\end{aligned}
$$

where

$$
G_{k, l}=e^{-\left(J_{x}+J_{y}+J_{x}^{\prime}+J_{y}^{\prime}\right) / 2} \sqrt{J_{x} J_{x}^{\prime}} K_{k, l}(\boldsymbol{J}) L_{k, l}(\boldsymbol{J}) .
$$

The kernel $G$ is again degenerated if the Hermitian expansion is truncated at a finite order. The eigenvalue equation for the $\pi$ mode is

$$
\operatorname{det}[M(\lambda)-I]=0,
$$

where $I$ is the identity matrix and

$$
M_{k, l ; k^{\prime}, l^{\prime}}(\lambda)=\int d \boldsymbol{J} \frac{J_{x} e^{-J_{x}-J_{y}}}{\lambda-Q(\boldsymbol{J})} L_{k, l}(\boldsymbol{J}) K_{k^{\prime}, l^{\prime}}(\boldsymbol{J})
$$

The eigenvalue of Eq. (41) is plotted in Fig. 1 as a function of the aspect ratio for two cases of $k_{\max }=1,2$. Two curves, close to each other, are drawn for each $k_{\max }$. They are from different methods of truncation of Hermitian expansion. The ones above come from square truncation ( $k \leq k_{\max }$ and $l \leq k_{\max }$ ), and the ones below from triangular truncation $\left(k+l \leq k_{\max }\right)$. The triangular truncation 


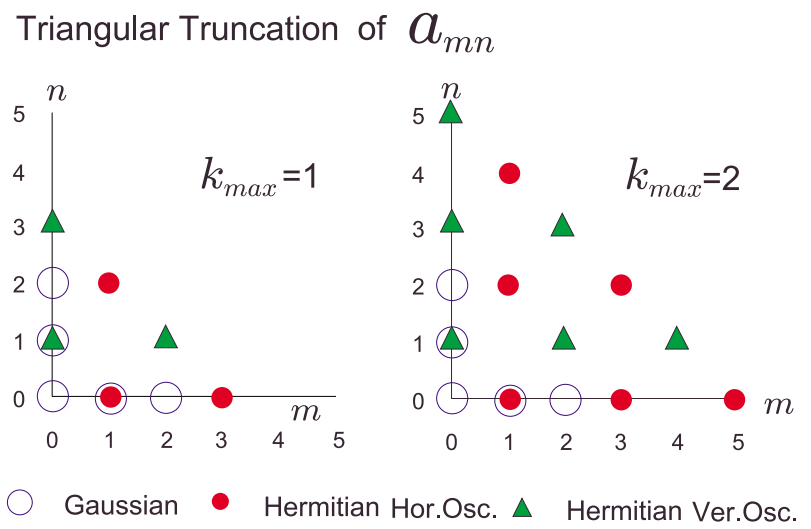

FIG. 4. (Color) The index of $a_{m n}$ to be taken in the triangular truncation for $k_{\max }=1$ and 2 . The open circles indicate the terms already taken in the soft-Gaussian approximation, and the filled circles (triangles) indicate the terms needed to describe horizontal (vertical) oscillations in the triangular truncation.

is illustrated in Fig. 4, which shows the index range of $a_{m n}$ to be taken for the triangle truncation for $k_{\max }=1,2$.

Some of the numerical values for round and very flat beams are listed in Table I. The numbers in brackets for $k_{\max }=1$ and 2 are from square truncation, others from triangular truncation.

One finds the following: (i) the eigenvalue approaches the value of exact theory as $k_{\max }$ increases; (ii) The difference between square and triangular truncations is extremely small (therefore, triangular truncation is better in practice due to the smaller number of terms); and (iii) $k_{\max }=2$ (fifth order polynomial times Gaussian distribution) already gives satisfactory results. Even $k_{\max }=1$ (third order polynomial) may be enough depending on the purpose.

As described in the Appendix, the function $K_{k, l}(J)$ is a simple elementary function of $\boldsymbol{J}$ when $R=0$ so that

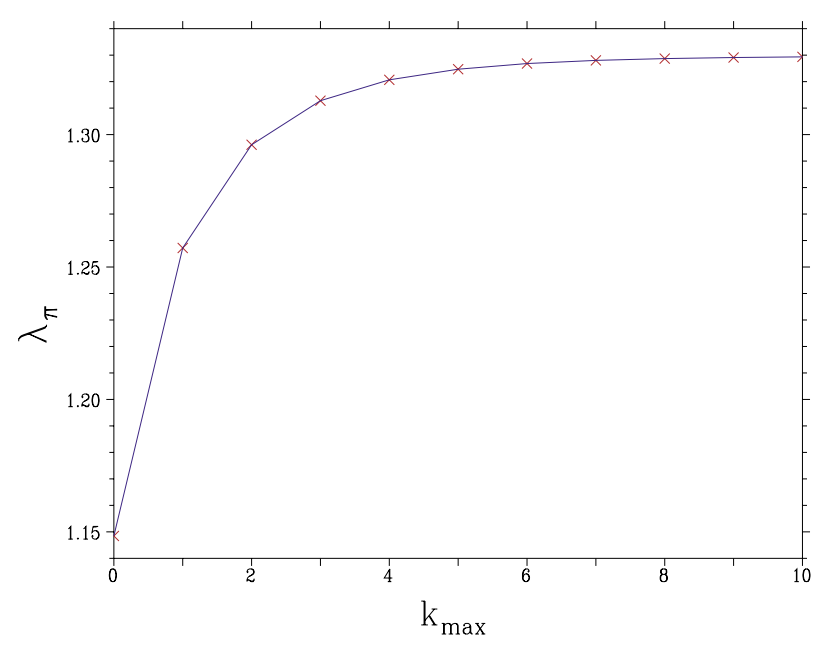

FIG. 5. (Color) The first $\pi$-mode eigenvalue for $R=0$ as a function of the truncation of Hermitian expansion.

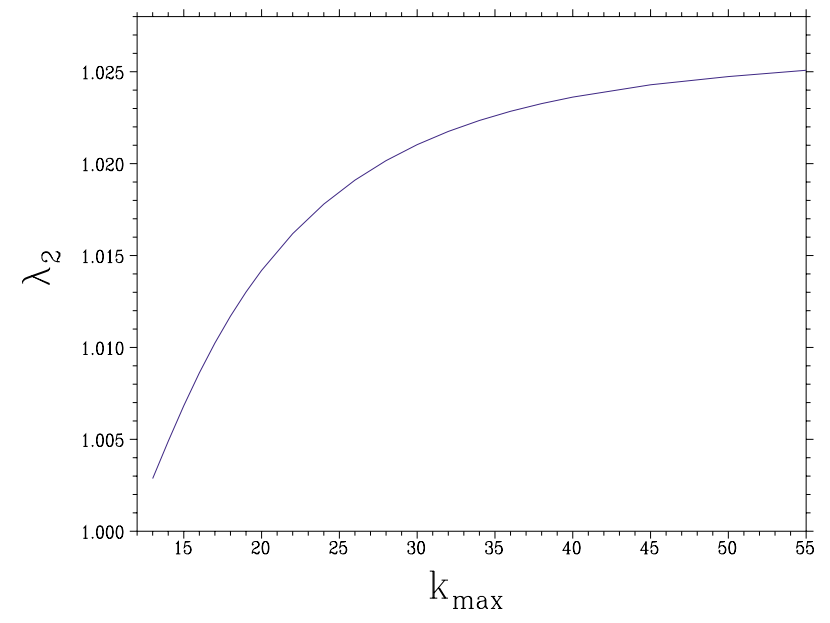

FIG. 6. (Color) The second $\pi$-mode eigenvalue for $R=0$ as a function of the truncation of Hermitian expansion.

higher order can easily be computed. To see the convergence with respect to $k_{\max }$ the eigenvalues for large $k_{\max }$ for $R=0$ were computed and plotted in Fig. 5. The approach to the limit is exponential with $k_{\max }$ rather than polynomial.

When $k_{\max }$ is large enough, one also finds the second eigenvalue close to the continuum, which was first found by Alexahin [13]. It is plotted in Fig. 6 as a function of $k_{\max }$ for the case $R=0$. It converges to the value 1.026 found in [13] although the convergence is very slow.

\section{CONCLUSIONS}

We have studied the nature of the soft-Gaussian approximation for beam-beam simulation with respect to the ratio $\lambda$ of the coherent oscillation frequency shift to the incoherent and found that (i) the soft-Gaussian approximation gives somewhat lower $\lambda$, namely, $\lambda_{\text {soft-Gaussian }} \approx$ $\left(1+\lambda_{\text {exact }}\right) / 2$, (ii) this might, in some cases, cause the Landau damping to occur more easily than it should, and (iii) this can be cured by using a distribution of the form Hermitian polynomials times Gaussian.

Since the soft-Gaussian approximation is a very useful tool for studying the strong-strong interaction, in particular that of hadron beams, it is highly desired to confirm its validity in other respects.

\section{ACKNOWLEDGMENT}

The author thanks Dr. Zimmermann for helpful discussions and information.

\section{APPENDIX}

We summarize some mathematical expressions used in the text.

First, the polynomial $L_{k, l}(\boldsymbol{J})$ introduced in Eq. (35) is defined by 


$$
\begin{aligned}
L_{k, l}(\boldsymbol{J})= & \frac{1}{(2 k+1) !(2 l) !} \int \frac{d \phi_{x} d \phi_{y}}{(2 \pi)^{2}} \frac{2 \cos \phi_{x}}{\sqrt{2 J_{x}}} \\
& \times H_{2 k+1}\left(\sqrt{2 J_{x}} \cos \phi_{x}\right) H_{2 l}\left(\sqrt{2 J_{y}} \cos \phi_{y}\right) \\
\equiv & L_{x, k}\left(J_{x}\right) L_{y, l}\left(J_{y}\right) .
\end{aligned}
$$

Here, $L_{x, k}\left(L_{y, l}\right)$ is a polynomial of $J_{x}\left(J_{y}\right)$ of order $k(l)$ and is given explicitly by

$$
\begin{gathered}
L_{x, k}=\frac{1}{2^{k}} \sum_{i=0}^{k} \frac{(-1)^{k-i}}{i !(i+1) !(k-i) !} J_{x}^{i}, \\
L_{y, l}=\frac{1}{2^{l}} \sum_{j=0}^{l} \frac{(-1)^{l-j}}{(j !)^{2}(l-j) !} J_{y}^{j} .
\end{gathered}
$$

The first few of them are

$$
\begin{gathered}
L_{x, 0}=1, \quad L_{x, 1}=\frac{1}{4} J_{x}-\frac{1}{2}, \\
L_{x, 2}=\frac{1}{48} J_{x}^{2}-\frac{1}{8} J_{x}+\frac{1}{8}, \\
L_{y, 0}=1, \quad L_{y, 1}=\frac{1}{2} J_{y}-\frac{1}{2}, \\
L_{y, 2}=\frac{1}{16} J_{y}^{2}-\frac{1}{4} J_{y}+\frac{1}{8} .
\end{gathered}
$$

The function $K_{k, l}(\boldsymbol{J})$ defined in Eq. (37) is obtained by the integration

$$
\begin{aligned}
\frac{K_{k, l}(\boldsymbol{J})}{1+R}= & \frac{\partial}{\partial J_{x}} \int \frac{d \phi}{(2 \pi)^{2}} \int d x^{\prime} d y^{\prime} H_{2 k}\left(x^{\prime}\right) H_{2 l}\left(y^{\prime}\right) \\
& \times \log \left[\left(x-x^{\prime}\right)^{2}+R^{2}\left(y-y^{\prime}\right)^{2}\right] \\
& \times \frac{e^{-\left(x^{\prime 2}+y^{\prime 2}\right) / 2}}{2 \pi} .
\end{aligned}
$$

In the case $R=0$, only $K_{k, 0}$ is relevant and is given explicitly by

$$
\begin{aligned}
K_{k, 0}= & \frac{\delta_{k, 0}}{J_{x}}-\frac{e^{-J_{x}}}{J_{x}} \sum_{j=0}^{k} \sum_{r=j}^{k} \\
& \times \frac{(-1)^{k-r}(2 k) !(2 r-2 j-1) ! !}{2^{r-j}(2 k-2 r) ! !(2 r-1) ! !(r-j) !} \frac{J_{x}^{j}}{j !} .
\end{aligned}
$$

The first few are

$$
\begin{gathered}
K_{0,0}=Q=\left(1-e^{-J_{x}}\right) / J_{x}, \\
K_{1,0}=-2 e^{-J_{x}}, \quad K_{2,0}=e^{-J_{x}}\left(8-4 J_{x}\right) .
\end{gathered}
$$

In general cases one has to obtain by numerical integrations (or by Taylor expansion for small $\boldsymbol{J}$ ). The most convenient form for numerical integration is presumably

$$
\begin{aligned}
K_{k, l}= & \frac{1+R}{\pi} \int_{0}^{2 \pi} d \theta \\
& \times \frac{(-1)^{k+l} \cos ^{2 k+2} \theta \sin ^{2 l} \theta}{R \cos ^{2} \theta+R^{-1} \sin ^{2} \theta} \\
& \times A_{k+l}\left(\sqrt{2 J_{x}} \cos \theta, \sqrt{2 J_{y}} \sin \theta\right),
\end{aligned}
$$

where

$$
A_{n}(\alpha, \beta)=\int_{0}^{\infty} d \omega \omega^{2 n} \frac{J_{1}(\alpha \omega)}{\alpha} J_{0}(\beta \omega) e^{-\omega^{2} / 2} .
$$

$A_{n}(n \geq 1)$ can be expressed by the modified Bessel functions

$$
A_{n}(\alpha, \beta)=\left[f_{n} I_{0}(\alpha \beta)-g_{n} I_{1}(\alpha \beta)\right] \frac{e^{-\left(\alpha^{2}+\beta^{2}\right) / 2}}{\alpha},
$$

where $f_{n}$ and $g_{n}$ are polynomials of $\alpha$ and $\beta$. The first few of them are

$$
\begin{array}{ll}
f_{1}=\alpha, & g_{1}=\beta, \quad f_{2}=4 \alpha-\alpha^{3}-3 \alpha \beta^{2}, \\
& g_{2}=2 \beta-3 \alpha^{2} \beta-\beta^{3} .
\end{array}
$$

$A_{0}$ cannot be written in the form Eq. (A11). Instead, for $K_{0,0}(\boldsymbol{J})=Q(\boldsymbol{J})$ we have the formula

$$
\begin{aligned}
Q(J)= & \int_{0}^{1} d t \frac{1+R}{\sqrt{R^{2}-1+1 / t^{2}}} \\
& \times\left[\tilde{I}_{0}\left(\frac{t^{2}}{2} J_{x}\right)-\tilde{I}_{1}\left(\frac{t^{2}}{2} J_{x}\right)\right] \\
& \times \tilde{I}_{0}\left(\frac{1}{2} \frac{R^{2}}{R^{2}-1+1 / t^{2}} J_{y}\right),
\end{aligned}
$$

where $\tilde{I}_{n}(x)=e^{-x} I_{n}(x)$.

[1] R. E. Meller and R.H. Siemann, IEEE Trans. Nucl. Sci. 28, 2431 (1981).

[2] K. Yokoya and H. Koiso, Part. Accel. 27, 181 (1990).

[3] H. Koiso et al., Part. Accel. 27, 83 (1990).

[4] M. Tawada, Y. Funakoshi, M. Masuzawa, and K. Ohmi, in Proceedings of the European Particle Accelerator Conference, 2000, Vienna, Austria (to be published), p. 1193.

[5] E. A. Perevedentsev and A. A. Valishev, in Proceedings of the European Particle Accelerator Conference, 2000, Vienna, Austria (to be published), p. 1223.

[6] K. Ohmi, Phys. Rev. E 62, 7287 (2000).

[7] M. P. Zorzano and F. Jones (to be published).

[8] M.P. Zorzano "Numerical integration of the Vlasov equation of two colliding beams" (to be published). 
[9] E. Keil, CERN LEP Note No. 294, 1981.

[10] M. P. Zorzano and F. Zimmermann, Phys. Rev. ST Accel. Beams 3, 044401 (2000); in Proceedings of the European Particle Accelerator Conference, 2000, Vienna, Austria (to be published), p. 1229.

[11] M. A. Furman, Report No. LBNL-45363/CBP Note-333,
2000.

[12] K. Hirata, Nucl. Instrum. Methods Phys. Res. Sect. A 269, 7 (1988).

[13] Y. Alexahin, Part. Accel. 59, 43 (1998).

[14] M. Bassetti and G. Erskine, Report No. CERN ISR TH/80-06, 1980. 